\title{
Acute Leukoencephalopathy with Restricted Diffusion
}

\author{
Mahesh Kamate \\ Department of Pediatrics, JN Medical College, KLE University, Belgaum, Karnataka, India
}

\section{Abstract}

Treatment and outcome of children with acute encephalopathy depend on the cause, prompt treatment of the underlying cause, and use of adequate supportive measures. Many novel causes of acute encephalopathy are emerging where lumbar puncture, computed tomography of the head, and routine biochemical testing can be normal such as acute disseminated encephalomyelitis and febrile infection-related refractory epilepsy syndrome. Magnetic resonance imaging (MRI) plays an important role in the workup of children with acute leukoencephalopathy. Despite this in few cases, a correct diagnosis is not possible and novel conditions have been described in the last decade. One such condition is acute encephalopathy with biphasic seizures and restricted diffusion also called as acute leukoencephalopathy with restricted diffusion. Here, the routine MRI sequences such as T1, T2, and fluid-attenuated inversion recovery sequences can be normal. Here, we have reviewed the etiology, types, clinicoradiological features, and treatment of this condition.

Keywords: Acute encephalopathy, acute encephalopathy with biphasic seizures and restricted diffusion, magnetic resonance imaging brain Acute Leukoencephalopathy with Restricted Diffusion

\section{Acute Leukoencephalopathy with Restricted DifFusION}

Acute encephalopathy refers to acute insult to the brain and presents with seizures, impaired consciousness, and other neurological symptoms. Acute encephalopathy as a complication of common viral (rarely bacterial) infections often affects young children and causes death or severe neurological sequelae. ${ }^{[1]}$ A correct diagnosis helps in institution of appropriate treatment and most importantly prognosis. The common causes of acute encephalopathy include febrile encephalopathy (viral meningoencephalitis, bacterial meningitis, tubercular meningitis, cerebral malaria, and acute disseminated encephalomyelitis [ADEM]), systemic disorders (dyselectrolytemia, hepatic/renal failure, and sepsis), hypoxic-ischemic encephalopathy, toxins, poisoning, traumatic brain injury, and stroke. In the last two decades, many new entities that cause acute encephalopathy in children have been described such as ADEM, acute necrotizing encephalitis (ANE), fever-induced refractory epilepsy syndrome (FIRES), clinically Mild encephalopathy with reversible splenial lesion (MERS), and autoimmune encephalitis..$^{[2,3]}$

Recently, in the last decade, a new viral-associated encephalopathy, acute encephalopathy with biphasic seizures

\begin{tabular}{|l|l|}
\hline \multicolumn{3}{c|}{ Access this article online } \\
\hline Quick Response Code: & Website: \\
& www.ijccm.org \\
\hline
\end{tabular}

and restricted diffusion (AESD), has been described mainly from the Asian continent. ${ }^{[4]}$ As the name suggests, this entity is characterized by a prolonged seizure with fever for 1-2 days followed by spontaneous improvement and this followed by worsening of clinical state with cluster of seizures, altered sensorium 3-4 days later. The second phase is characterized by areas of restricted diffusion on the magnetic resonance imaging (MRI). Soon, there were reports of children presenting with acute encephalopathy, seizures, and restricted areas of diffusion on MRI at presentation itself but without the characteristic biphasic clinical picture. They had relatively severe clinical course and poor prognosis. ${ }^{[1]}$ The label of AESD is not appropriate for this group. Presentation as acute encephalopathy, restricted areas of diffusion seen in both gray and white matter that is the hallmark of this condition, makes use of the term acute leukoencephalopathy with restricted diffusion (ALERD) more appropriate for this condition. Those occurring in the setting of an infection can be termed as infectious ALERD as similar presentation is seen with toxins such as cranial irradiation, therapeutic agents, and

Address for correspondence: Dr. Mahesh Kamate, Department of Pediatrics, JN Medical College, KLE University, Belgaum - 590 010, Karnataka, India. E-mail:drmaheshkamate@gmail.com

This is an open access journal, and articles are distributed under the terms of the Creative Commons Attribution-NonCommercial-ShareAlike 4.0 License, which allows others to remix, tweak, and build upon the work non-commercially, as long as appropriate credit is given and the new creations are licensed under the identical terms.

For reprints contact: reprints@medknow.com

How to cite this article: Kamate M. Acute leukoencephalopathy with restricted diffusion. Indian J Crit Care Med 2018;22:519-23. 
drugs of abuse and environmental toxins where the term toxic ALERD is preferred. ${ }^{[5]}$ We review the existing literature on this infectious ALERD in this article.

\section{Acute Leukoencephalopathy With Restricted Diffusion}

It is a clinical-radiological syndrome and is one of the infection-associated encephalopathy syndromes seen in childhood. It has a low mortality rate but has a higher incidence $\left(>2 / 3^{\text {rd }}\right)$ of neurologic sequelae. It has been reported mainly from East Asia. In a series of 44 patients from Nagoya University, Japan, causative pathogens isolated were human herpesvirus-6, adenovirus, rotavirus, influenza virus, Mycoplasma pneumoniae, Enterovirus type Coxsackievirus A6, Escherichia coli O157:H7, and Streptococcus pneumonia. ${ }^{[6]}$ At times, it can be a presentation of bacterial septic encephalopathy like S. pneumonia ${ }^{[7]}$

\section{Clinical Features}

Presentation is mainly in the form of acute encephalopathy associated with fever. There are varying degrees of altered sensorium and usually a prolonged seizure ( $>30 \mathrm{~min}$ ) on the $1^{\text {st }}$ day. The seizure could be generalized or partial and could be recurrent. There are reports of brief seizures lasting for few minutes only, and sometimes, it could be in the form of nonconvulsive seizure also. ${ }^{[5]}$

\section{TyPeS OF ACUTE LeUKOENCEPHALOPATHY WITH Restricted Diffusion}

Two distinct groups according to the distribution of brain lesions have been identified. The one with diffuse areas of restricted diffusion on MRI is called diffuse ALERD and those where there is sparing of the central regions of the brain are called as central-sparing ALERD. The latter is equivalent to the well-described and well-known acute encephalopathy with biphasic seizures and late reduced diffusion (AESD). ${ }^{[1]}$

\section{Diffuse Acute Leukoencephalopathy with Restricted Diffusion}

This type is associated with a severe phenotype with rapid and severe deterioration of consciousness. Coma is observed within $24 \mathrm{~h}$ after onset of disease. A biphasic clinical course characteristic of AESD is rare. Seizures at the onset of the disease are not always present. The outcome is poor and can be associated with mortality. Sequelae in the form of cognitive impairment, epilepsy, and behavioral problems are known. A systemic inflammatory response in which multiple organ failure, shock, and disseminated intravascular coagulation are often observed in patients with ALERD. Because of varying prognosis, it is important to make this distinction at presentation.

\section{Central-Sparing Lesions/AESD}

This is a relatively mild phenotype of acute encephalopathy. A biphasic clinical course is a characteristic of this group of patients. Onset is often marked by a prolonged seizure ( $>30 \mathrm{~min})$ with fever followed by improved consciousness. Clustered seizures, signs of frontal lobe dysfunction, and worsening of consciousness become apparent at 3-4 days after onset. Coma is uncommon and various degrees of cognitive impairment are observed as neurologic sequelae.

\section{Pathogenesis}

Most patients show hypercytokinemia in the form of elevated interleukin-6 (IL-6), IL-8, and IL-10 and tumor necrosis factor in the serum and IL-4, IL-6, and IL-8 in the cerebrospinal fluid (CSF). Excitotoxic injury with delayed (or apoptotic) neuronal death is hypothesized as a possible mechanism. There are reports of cases of ALERD associated with hemophagocytic syndrome, thereby suggesting the role of hypercytokinemia as pathogenesis of ALERD. ${ }^{[8]}$ MR spectroscopy in children with ALERD usually shows decreased N-acetylaspartate (NAA) and elevated glutamine/glutamate complex (Glx) during the week of presentation. Afterward, Glx normalizes, whereas NAA remains low in children with neurologic sequelae but normalizes in those without neurologic sequelae. ${ }^{\left[{ }^{[]}\right.}$These findings support the hypothesis that excitotoxic neuronal damage plays an important role in the pathogenesis of ALERD and suggest that MR spectroscopy might be predictive of outcome.

Unlike ADEM/autoimmune encephalitis, in ALERD, no autoantibodies have been found, and it is not thought to be an immune-mediated disorder. Diffusion abnormalities are the result of glutamate-induced astrocytic swelling and edema. The pathophysiological mechanism of ALERD does not require direct infection of the central nervous system. Certain genetic predisposition factors for ALERD include certain genotype of carnitine palmitoyltransferase-II, SCN1A, SCN2A, ADORA2A, and TLR3. ${ }^{[10,11]}$

\section{Laboratory Abnormalities}

In diffuse ALERD, increased liver enzymes, hyperglycemia, hyperCKemia, and metabolic acidosis are prominent, while in central-sparing ALERD/AESD, these laboratory abnormalities are mild, if present. ${ }^{[1]}$ In diffuse ALERD, serum and CSF levels of inflammatory cytokines, such as IL-6 and tumor necrosis factor, are markedly elevated. The CSF examination is usually normal (no pleocytosis or increase in proteins). This finding helps in ruling out the infectious causes of acute febrile encephalopathy.

\section{NeURoIMAgING}

As the name indicates, ALERD is characterized by restricted diffusion on MRI and neuroimaging plays an important role in the diagnosis. ${ }^{[1]}$ Computed tomography (CT) scan of the brain 
is mostly normal unless late in the disease in severe cases, wherein there may be white matter hypodensities. Diagnosis is made on the MRI of the brain. Signal changes more pronounced on diffusion-weighted image (DWI) sequences. There are markedly restricted diffusion and edematous changes in the entire cortical and subcortical areas which promptly revert on apparent diffusion coefficient (ADC) maps [Figure 1]. T1-weighted (T1-W) image may show mild thickening of the cortex and T2-W/fluid-attenuated inversion recovery (FLAIR) sequences show mild hyperintensities in the cortex and white matter. Blurring of gray-white matter interface may also be seen. The changes on conventional sequences such as T1-W, T2-W, and FLAIR may be subtle, and unless DWIs are acquired, a diagnosis of ALERD can be easily missed. Thus, as clinicians when we ask for MRI in children with acute encephalopathy and when ALERD is suspected, we should inform the radiologist to include DWI with ADC map in the MRI protocol.

DW MRI provides potentially unique information on the viability of brain tissue. It provides image contrast that is dependent on the molecular motion of water, which may be substantially altered by disease. Both DWI and the corresponding ADC maps have to be studied to identify areas with restricted diffusion. Lesions that appear bright on DWI and
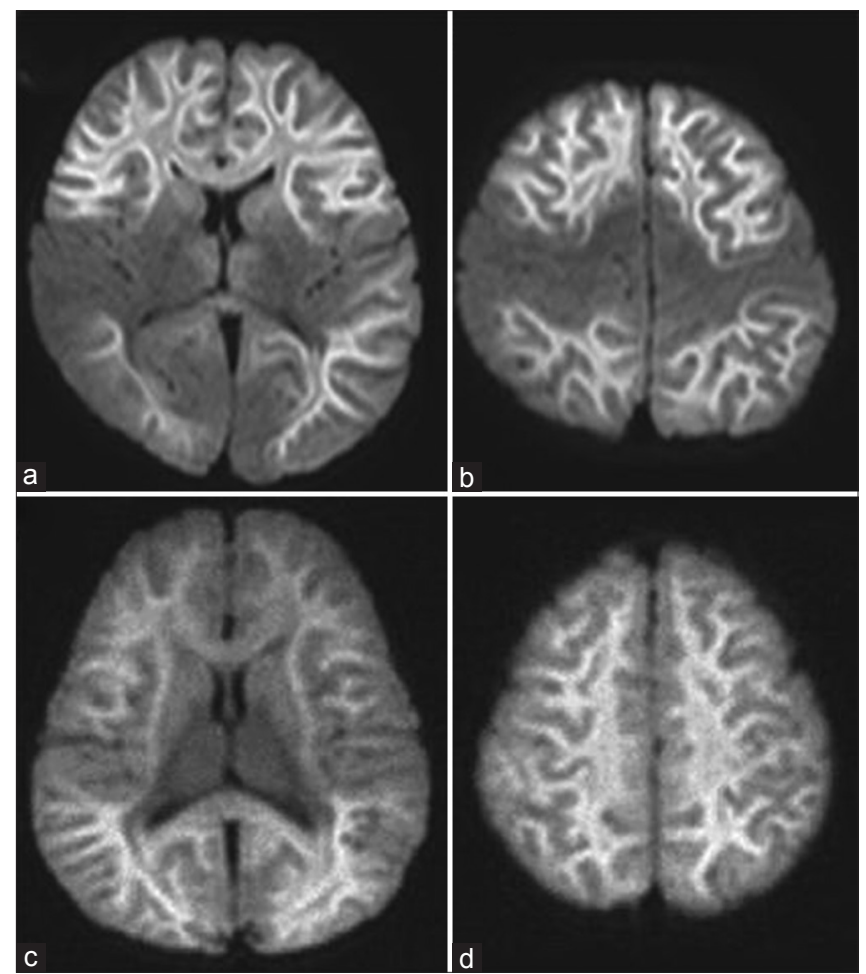

Figure 1: Diffusion-weighted image of acute leukoencephalopathy with restricted diffusion. ( $a$ and $b$ ) Central-sparing acute leukoencephalopathy with restricted diffusion shows sparing of the primary sensory motor cortex; (c and d) in diffuse acute leukoencephalopathy with restricted diffusion, there is diffuse bilateral involvement of the white matter. The white matter shows "bright tree appearance," which represents high-signal intensity on diffusion-weighted image in the widespread subcortical white matter, which has the appearance of tree branches turn dark on ADC map are mostly pathogenic. ${ }^{[5]}$ Lesions that are bright on T2-W image and FLAIR may sometime appear bright on DWI, but there would not be reversal on ADC map.

MRI of the brain shows the characteristic "bright tree appearance," which represents high-signal intensity on DWI in the widespread subcortical white matter, which has the appearance of tree branches [Figure 1]. It is important to note that MRI can be normal in the first 2-3 days, especially in the central-sparing ALERD, and in such cases, it is prudent to repeat MRI after day-4 of illness. Children with diffuse ALERD can show changes on MRI in the first few days.

Central sparing is defined as a lack of high-signal intensity on DWI in subcortical white matter in the primary sensorimotor areas, while diffuse ALERD involves both the hemispheres in a widespread manner. ${ }^{[1]}$ In those with diffuse ALERD also, there could be involvement of frontal and occipital regions in the initial few days, but this involvement quickly spreads to other areas by the end of 1 week and the restricted diffusion is then seen in the entire brain [Figure 1].

In patients with central-sparing ALERD, the areas without reduced diffusion were strictly limited, around the Sylvian fissures. Involvement of lateral areas in central-sparing ALERD is more prominently seen than those the medial regions. However, in diffuse ALERD, lesions were located in the anterior half of the frontal lobes and mesial areas of the occipital lobes. ${ }^{[1]}$

\section{FoLLOW-UP IMAGING}

There is mild-to-severe cerebral atrophy when the imaging is repeated after 2-4 weeks, and there is resolution of white matter restriction on DWI over 2-4 weeks. Laminar necrosis and increased signal intensities in the subcortical white matter on T2-W images in diffuse lesions can be seen. Hyperintensities can be seen in the caudate nucleus in some patients, whereas rest of basal ganglia, thalami, and corpus callosum are usually spared in ALERD.

\section{Differential Diagnosis}

Toxic leukoencephalopathy seen in patients exposed to a wide variety of agents such as cranial irradiation, therapeutic agents (antibiotics such as metronidazole, immunosuppressive drugs), and drugs of abuse and environmental toxins (carbon monoxide) forms close differentials for infectious ALERD which can be ruled out by careful history taking. Ischemia/infarction and encephalitis can also mimic ALERD, but the distribution of lesions and the CSF examination help in differentiating them from ALERD. ${ }^{[5]}$

Posterior reversible encephalopathy (PRES) and ADEM may also show focal restriction of diffusion in some cases, but the changes are more pronounced on T2-W images/FLAIR than on DWI unlike in ALERD ${ }^{[5]}$ Because of demyelination and increased water in the extravascular spaces, MRI in ADEM cases have ADCs higher than those of normal white matter. The 
lesions in ADEM are mostly multifocal with T2 abnormality that is best seen in T2-W/FLAIR images. In addition, the lesions are moderate to large, usually asymmetric, and patchy involving deep and subcortical white matter of cerebral, cerebellar hemisphere, and brainstem. In PRES, changes are prominent in the posterior regions and diffusion restriction occurs only in a minority of patients. The lesions are usually punctate and surrounded by much larger areas of edema.

The MRI in conditions such as FIRES is usually normal in initial few days to weeks. However, with ongoing seizures, signal changes may be seen in gray matter structures such as the mesial temporal lobes and hippocampi. ${ }^{[12]}$ In autoimmune encephalitis, MRI is either normal or shows involvement of the limbic structures with the involvement of the striatum, diencephalon, or rhombencephalon in some cases. ${ }^{[13]}$ In mild leukoencephalopathy with reversible splenial lesion (MERS), as the name suggests, the lesions are restricted to the splenium of corpus callosum and at times may extend into white matter. Furthermore, clinically, the illness is mild. ${ }^{[3]}$ MERS and ALERD could be parainfectious illness along the same spectrum but with varying severity. Another recently described parainfectious condition that presently acutely is ANE. Here, the CT/MRI shows characteristic signal changes in the thalami bilaterally which may later go for necrosis and hemorrhages may appear in the thalami. There may be involvement of brainstem, cerebellum, and white matter in ANE, but the thalami which are swollen in ANE help differentiating ALERD from ANE. ${ }^{[2]}$

\section{TREATMENT}

Intravenous (IV) pulse steroids and IV immunoglobin $\mathrm{G}$ are used after neuroinfections have been ruled out. ${ }^{[1]}$ There is no evidence for the same, and hence, there is a need to collect cases of ALERD and conduct randomized studies. As the proposed pathogenesis for ALERD is excitotoxic injury with delayed neuronal death, there have been some case reports with combination therapy of N-methyl-D-aspartate receptor antagonist, dextromethorphan, and apoptosis inhibitor, cyclosporine A. ${ }^{[14]}$ A study on four patients with ALERD showed favorable results. Other agents reported as potentially effective for ALERD include fosphenytoin, L-carnitine and vitamins, and erythropoietin. ${ }^{[15]}$

\section{Outcome}

In diffuse ALERD, up to 5\%-10\% mortality has been reported, while in the central-sparing ALERD/AESD, there have been no reports of death. More than $2 / 3^{\text {rd }}$ of children with ALERD develop sequelae. Postencephalopathic epilepsy is seen in $1 / 4^{\text {th }}$ of children. ${ }^{[4]}$ Other long-term sequelae include hyperactivity with language delay, cognitive impairment, spasticity, dyskinesias, and hemiparesis.

\section{Postencephalopathic Epilepsy}

The reported incidence is $23 \%$ and the median interval between the onset of ALERD and occurrence of postencephalopathic epilepsy is 8.5 months. ${ }^{[4]}$ Epileptic spasms and startle focal seizures are common seizure types. Other seizures types include the myoclonic, dyscognitive, and tonic type. Few have seizures that are induced by sudden unexpected sounds. Seizure types confirmed by ictal video-electroencephalography recordings are epileptic spasms and focal seizures with frontal onset, and all focal seizures are startle seizures induced by sudden acoustic stimulation. The seizure types may be determined by the diffuse subcortical white matter injury and age-dependent reorganization of the brain network. Occipital lesions were associated with the earliest onset of spasms and frontal lesions were associated with the latest onset. Patients with postencephalopathic epilepsy have more severe cognitive impairments than those without it and it is usually intractable. The biphasic seizures and status epilepticus that occur during the acute phase of encephalopathy do not influence the risk of postencephalopathic epilepsy.

As the clinicoradiological presentation of ALERD resembles other acute encephalopathy syndromes, such as acute infantile encephalopathy predominantly affecting the frontal lobes, acute encephalopathy of obscure origin, and hemiconvulsion-hemiplegia-epilepsy syndrome, it is likely that it is part of a spectrum of disorders that share a common final nonimmune pathophysiological process, resulting in cerebral injury with resultant atrophy. ${ }^{[16-18]}$

\section{Conclusion}

ALERD is a novel cause for acute encephalopathy in Pediatric Intensive Care Unit. Diagnosis is based on the finding of restricted diffusion in white matter and/or cortex on DWI in MRI of the brain. MRI may be normal in the first 3-4 days, and hence, it is prudent to repeat MRI after 5 days if ALERD is a strong suspect in a given case. Supportive care and prompt treatment of seizures are very important. Diffuse ALERD has poor prognosis as compared to the central-sparing type of ALERD. Postencephalopathic epilepsy is seen in a quarter of patients and is intractable.

\section{Financial support and sponsorship}

Nil.

\section{Conflicts of interest}

There are no conflicts of interest.

\section{References}

1. Okumura A, Kidokoro H, Tsuji T, Suzuki M, Kubota T, Kato T, et al. Differences of clinical manifestations according to the patterns of brain lesions in acute encephalopathy with reduced diffusion in the bilateral hemispheres. AJNR Am J Neuroradiol 2009;30:825-30.

2. Mizuguchi M, Abe J, Mikkaichi K, Noma S, Yoshida K, Yamanaka T, et al. Acute necrotising encephalopathy of childhood: A new syndrome presenting with multifocal, symmetric brain lesions. J Neurol Neurosurg Psychiatry 1995;58:555-61.

3. Tada H, Takanashi J, Barkovich AJ, Oba H, Maeda M, Tsukahara H, et al. Clinically mild encephalitis/encephalopathy with a reversible splenial lesion. Neurology 2004;63:1854-8.

4. Takanashi J, Oba H, Barkovich AJ, Tada H, Tanabe Y, Yamanouchi H, et al. Diffusion MRI abnormalities after prolonged febrile seizures with 
encephalopathy. Neurology 2006;66:1304-9.

5. Singh V, Tomar V, Kumar A, Phadke RV. Acute leucoencephalopathy with restriction of diffusion - A case report. Eastern J Med 2012;17:149-52.

6. Ito Y, Natsume J, Kidokoro H, Ishihara N, Azuma Y, Tsuji T, et al. Seizure characteristics of epilepsy in childhood after acute encephalopathy with biphasic seizures and late reduced diffusion. Epilepsia 2015;56:1286-93.

7. Yamaguchi H, Tanaka T, Maruyama A, Nagase H. Septic encephalopathy characterized by acute encephalopathy with biphasic seizures and late reduced diffusion and early nonconvulsive status epilepticus. Case Rep Neurol Med 2016;2016:7528238.

8. Tadokoro R, Okumura A, Nakazawa T, Hara S, Yamakawa Y, Kamata A, et al. Acute encephalopathy with biphasic seizures and late reduced diffusion associated with hemophagocytic syndrome. Brain Dev 2010;32:477-81.

9. Takanashi J, Tada H, Terada H, Barkovich AJ. Excitotoxicity in acute encephalopathy with biphasic seizures and late reduced diffusion. AJNR Am J Neuroradiol 2009;30:132-5.

10. Saitoh M, Shinohara M, Hoshino H, Kubota M, Amemiya K, Takanashi JL, et al. Mutations of the SCN1A gene in acute encephalopathy. Epilepsia 2012;53:558-64.

11. Saitoh M, Shinohara M, Ishii A, Ihara Y, Hirose S, Shiomi M, et al. Clinical and genetic features of acute encephalopathy in children taking theophylline. Brain Dev 2015;37:463-70.
12. Rivas-Coppola MS, Shah N, Choudhri AF, Morgan R, Wheless JW Chronological evolution of magnetic resonance imaging findings in children with febrile infection-related epilepsy syndrome. Pediatr Neurol 2016;55:22-9.

13. Kelley BP, Patel SC, Marin HL, Corrigan JJ, Mitsias PD, Griffith B, et al. Autoimmune encephalitis: Pathophysiology and imaging review of an overlooked diagnosis. AJNR Am J Neuroradiol 2017;38:1070-8.

14. Matsuo M, Maeda T, Ono N, Sugihara S, Kobayashi I, Koga D, et al. Efficacy of dextromethorphan and cyclosporine a for acute encephalopathy. Pediatr Neurol 2013;48:200-5.

15. Nakazawa M, Akasaka M, Hasegawa T, Suzuki T, Shima T, Takanashi J, et al. Efficacy and safety of fosphenytoin for acute encephalopathy in children. Brain Dev 2015;37:418-22.

16. Yamanouchi H, Kawaguchi N, Mori M, Imataka G, Yamagata T, Hashimoto $\mathrm{T}$, et al. Acute infantile encephalopathy predominantly affecting the frontal lobes. Pediatr Neurol 2006;34:93-100.

17. Maegaki Y, Kondo A, Okamoto R, Inoue T, Konishi K, Hayashi A, et al. Clinical characteristics of acute encephalopathy of obscure origin: A biphasic clinical course is a common feature. Neuropediatrics 2006;37:269-77.

18. Freeman JL, Coleman LT, Smith LJ, Shield LK. Hemiconvulsion-hemiplegia-epilepsy syndrome: Characteristic early magnetic resonance imaging findings. J Child Neurol 2002;17:10-6. 